\title{
Deformation Measuring Methods Based on Inertial Sensors for Airborne Distributed POS
}

\author{
Xiaolin Gong, ${ }^{1,2}$ Haojie Liu, ${ }^{1,2}$ and Xing-Gang Yan $^{3}$ \\ ${ }^{1}$ School of Instrumentation Science and Optoelectronics Engineering, Beihang University, Beijing 100191, China \\ ${ }^{2}$ Science and Technology on Inertial Laboratory, Beijing 100191, China \\ ${ }^{3}$ School of Engineering and Digital Arts, University of Kent, Canterbury CT2 7NT, UK
}

Correspondence should be addressed to Xiaolin Gong; gongxiaolin@buaa.edu.cn

Received 25 May 2017; Accepted 2 August 2017; Published 10 September 2017

Academic Editor: Nicolas Avdelidis

Copyright (C) 2017 Xiaolin Gong et al. This is an open access article distributed under the Creative Commons Attribution License, which permits unrestricted use, distribution, and reproduction in any medium, provided the original work is properly cited.

\begin{abstract}
This paper is focused on deformation measuring methods based on inertial sensors, which are used to achieve high accuracy motion parameters and the spatial distribution optimization of multiple slave systems in the airborne distributed Position and Orientation System or other purposes. In practical application, the installation difficulty, cost, and accuracy of measuring equipment are the key factors that need to be considered synthetically. Motivated by these, deformation measuring methods based on gyros and accelerometers are proposed, respectively, and compared with the traditional method based on the inertial measurement unit (IMU). The mathematical models of these proposed methods are built, and the detailed derivations of them are given. Based on the Kalman filtering estimation, simulation and semiphysical simulation based on vehicle experiment show that the method based on gyros can obtain a similar estimation accuracy to the method based on IMU, and the method based on accelerometers has an advantage in $y$-axis deformation estimation.
\end{abstract}

\section{Introduction}

The airborne distributed Position and Orientation System (POS) has been proposed to achieve multipoint spatiotemporal motion parameters for synthetical earth observation systems with multiple remote sensing loads [1-3]. Distributed POS can be composed of a few high precision master systems, some low precision slave systems, POS Computer System (PCS), and postprocessing software. Usually, the master system is a high precision integrated system of Strapdown Inertial Navigation System and Global Navigation Satellite System [4] (also called the main POS). The slave system is only an inertial measurement unit (IMU), which consists of three orthogonally mounted gyros and accelerometers, respectively, and is placed as close as possible to the location of the load. The slave systems, also called the sub-IMUs, depend on the master system to transfer alignment to achieve their high accuracy motion parameters. Due to the deformation of aircraft caused by gust, turbulence, and other factors, there is a time-varying and complex flexure angle between the main POS and each sub-IMU besides the rigid misalignment angle. The schematic diagram of the measuring system and the cross section of aircraft with deformation at a certain moment are shown in Figure 1, where the grey part with dotted line is the ideal state of the wings without any deformation. It is clear to see that the premise and key of high accuracy transfer alignment is the attitude transformation, determined by flexure and misalignment angle between the master system and slave system, which can be estimated and compensated with high accuracy.

Furthermore, when there are many remote sensing loads working simultaneously, airborne distributed array antenna Synthetic Aperture Radar (SAR) is a typical example which has many subantennas on both sides of the wing; the high accuracy motion parameters of each load must be measured $[5,6]$. Since the bearing capacity of aircraft is limited, especially the wing section, there are very stringent requirements on the weight and size of the measurement equipment, while the measurement accuracy of sub-IMU is proportional to the weight and size. It should be noted that a high accuracy sub-IMU may not be available at the location of each load, and the positions of sub-IMU and load are not 


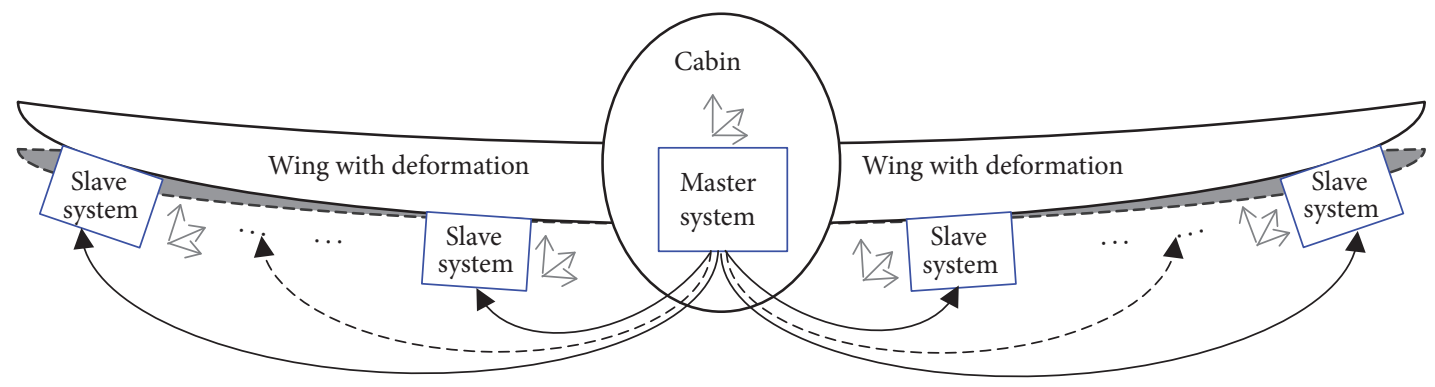

FIGURE 1: The schematic diagram of the measuring system and the cross section of aircraft.

always matched with each other. Therefore, it is necessary to consider the arrangement optimization of the distributed POS, such that the high precision motion parameters of all loads can be obtained using the minimum sub-IMUs. And the arrangement optimization also requires the attitude transformation between each node [7-9].

At present, aircraft deformation measuring methods can be summarized into three types: strain sensor measurement, optical measurement, and inertial measurement. The strain sensor measurement can be traced back to the 1940s and later it was improved by Skopinski et al. [10, 11]. It is a kind of mechanical measuring method which is widely used because of its convenient operation. However, it has limitation to the aircraft material and needs many wires which will increase the load of the aircraft. Besides, the strain sensor is easily affected by the physical abrasion, temperature, and so forth [12-14]. As for optical measurement, the Dutch National Aerospace Laboratory (NLR) used a camera to record the black and white striped pattern on the wing surface to estimate the flexible deformation [15]. Then, NLR presented a noncontact optical measurement which can obtain the deformation rule [16]. In addition, there are other optical measurements using visual sensors, optical fiber sensors, and bionic optical sensors to measure the flexible deformation [17-19]. All those optical measurements need the external measurement components and the beam transceivers must be intervisible, which make them not only complex to be installed, but also prone to be affected by the weather conditions. Inertial measurement is mainly based on the IMUs which are installed at the places of both main and subnodes. The difference of the navigation results between the main node and subnode, such as attitude difference and velocity difference, is utilized to estimate the flexible deformation. This procedure is known as the transfer alignment [20, 21].

Compared with IMU, three gyros or three accelerometers will reduce the weight, cost, and size of the measurement equipment. Particularly, high precision accelerometers have significant advantages of small size, light weight, low cost, and convenient installation when compared with the IMUs and gyros. It is necessary and significant to study the deformation measurement using gyros or accelerometers only. Therefore, this paper deduces the formulations of deformation measurement based on gyros (Gs) and accelerometers (As), respectively, and provides the mathematical modeling and algorithm design of deformation measuring method based on IMU, Gs, and As. Finally, taking the deformation measurement of the wing as an example, the measurement accuracies of these three methods are compared and analyzed by the simulation experiment based on the Kalman filter (KF).

The rest of this paper is organized as follows. In Section 2, the mathematical modeling of deformation measuring algorithms based on IMU, Gs, and As is given. In Section 3, detailed numerical simulation and semiphysical simulation are performed. Section 4 concludes this paper.

\section{Mathematical Modeling}

The detailed mathematical modeling of the three deformation measuring algorithms based on inertial sensors is given in this section. The details of KF can be found in [22, 23].

The measurement systems at the main node and the subnode can be called the master system and slave system, respectively. The coordinate frames used in this paper are defined as follows: $i$ and $e$ denote the earth-centered inertial frame and the earth-centered earth-fixed frame, respectively. The navigation frames of the master system and slave system (an IMU or three gyros or three accelerometers) are defined with $x-y$ - $z$-axes pointing to east-north-up (E-N-U), represented by $n_{m}$ and $n_{s}$, respectively. The body frames of the master system and slave system are defined on the rigid body of the inertial measurement unit and are denoted by $b_{m}$ and $b_{s}$, respectively. A detailed description of these coordinate frames is available in $[21,24,25]$.

2.1. Mathematical Model of Deformation Measuring Algorithm Based on IMU. This method needs three orthogonally mounted gyros and accelerometers, respectively, on each note. For distributed POS, the main POS can be used as the master system and the sub-IMU can be used as the slave system. The mathematical model for KF includes the state equation and measurement equation.

2.1.1. Error Equation. The state equation is based on the linear inertial navigation error equation of the sub-IMU, and the differential equation of the flexure angle and rigid misalignment angle is added.

Due to the existence of both flexure angle and rigid misalignment angle, the attitude transfer relationship between 
the master system and the slave system is shown as follows:

$$
\mathbf{C}_{b_{s}}^{b_{m}}=\left[\begin{array}{ccc}
1 & -u_{z} & u_{y} \\
u_{z} & 1 & -u_{x} \\
-u_{y} & u_{x} & 1
\end{array}\right]=I+\mathbf{u} \times
$$

where $\mathbf{C}_{b_{s}}^{b_{m}}$ is the coordinate transformation matrix from $b_{s^{-}}$ frame to $b_{m}$-frame, and it is an orthogonal matrix; system error angle $\mathbf{u}=\boldsymbol{\rho}+\boldsymbol{\theta}$, where $\boldsymbol{\rho}=\left[\begin{array}{lll}\rho_{x} & \rho_{y} & \rho_{z}\end{array}\right]^{\mathrm{T}}$ and $\boldsymbol{\theta}=\left[\begin{array}{lll}\theta_{x} & \theta_{y} & \theta_{z}\end{array}\right]^{\mathrm{T}}$ represent rigid misalignment angle and flexure angle between the master system and slave system, respectively.

The following are all the error equations based on the defined coordinate frames. Firstly, the inertial navigation error equation of the sub-IMU is given in (2), which includes attitude error equation, velocity error equation, position error equation, and inertial sensor constant error equation [26, 27]:

$$
\begin{aligned}
\dot{\boldsymbol{\phi}}^{n_{s}}= & -\boldsymbol{\omega}_{i n_{s}}^{n_{s}} \times \boldsymbol{\phi}^{n_{s}}+\delta \boldsymbol{\omega}_{i n_{s}}^{n_{s}}+\mathbf{C}_{b_{s}}^{n_{s}} \boldsymbol{\varepsilon}^{b_{s}}, \\
\delta \dot{\mathbf{V}}^{n_{s}}= & \mathbf{f}^{n_{s}} \times \boldsymbol{\phi}^{n_{s}}-\left(2 \delta \boldsymbol{\omega}_{i e}^{n_{s}}+\delta \boldsymbol{\omega}_{e n_{s}}^{n_{s}}\right) \times \mathbf{V}^{n_{s}} \\
& -\left(2 \boldsymbol{\omega}_{i e}^{n_{s}}+\boldsymbol{\omega}_{e n_{s}}^{n_{s}}\right) \times \delta \mathbf{V}^{n_{s}}+\mathbf{C}_{b_{s}}^{n_{s}} \nabla^{b_{s}}, \\
\delta \dot{L}= & \frac{\delta V_{\mathrm{N}}}{R_{\mathrm{M}}+H}-\frac{V_{\mathrm{N}}}{\left(R_{\mathrm{M}}+H\right)^{2}} \delta H, \\
\delta \dot{\lambda}= & \frac{\sec L}{R_{\mathrm{N}}+H} \delta V_{\mathrm{E}}+\frac{V_{\mathrm{E}} \sec L \tan L}{R_{\mathrm{N}}+H} \delta L \\
& -\frac{V_{\mathrm{E}} \sec L}{\left(R_{\mathrm{N}}+H\right)^{2}} \delta H, \\
\delta \dot{H}= & \delta V_{\mathrm{U}}, \\
\dot{\boldsymbol{\varepsilon}}_{c}^{b_{s}}= & 0, \\
\dot{\nabla}_{c}^{b_{s}}= & 0,
\end{aligned}
$$

where the subscripts E, N, and U represent east, north, and up, respectively; $\phi^{n_{s}}=\left[\begin{array}{lll}\phi_{\mathrm{E}} & \phi_{\mathrm{N}} & \phi_{\mathrm{U}}\end{array}\right]^{\mathrm{T}}$ is the attitude error vector in $n_{s}$-frame; $\omega_{i n_{s}}^{n_{s}}$ is the rotation velocity of the $n_{s}$-frame relative to the $i$-frame expressed in $n_{s}$-frame with error $\delta \boldsymbol{\omega}_{i n_{s}}^{n_{s}}$; $\mathrm{C}_{b_{s}}^{n_{s}}$ is the coordinate transformation matrix from $b_{s}$-frame to $n_{s}$-frame; $\varepsilon^{b_{s}}$ is the gyro random drift of the slave system in $b_{s}$-frame, which consists of random constant drift $\varepsilon_{c}^{b_{s}}$ and Gaussian white noise $\boldsymbol{\omega}_{\varepsilon}^{b_{s}}[28,29]$ with $\boldsymbol{\varepsilon}_{c}^{b_{s}}=\left[\begin{array}{ccc}\varepsilon_{x}^{b_{s}} & \varepsilon_{y}^{b_{s}} & \varepsilon_{z}^{b_{s}}\end{array}\right]^{\mathrm{T}}$ and $\boldsymbol{\omega}_{\varepsilon}^{b_{s}}=\left[\begin{array}{lll}\omega_{\varepsilon_{x}}^{b_{s}} & \omega_{\varepsilon_{y}}^{b_{s}} & \omega_{\varepsilon_{z}}^{b_{s}}\end{array}\right]^{\mathrm{T}} ; \mathbf{V}^{n_{s}}=\left[\begin{array}{lll}V_{\mathrm{E}} & V_{\mathrm{N}} & V_{\mathrm{U}}\end{array}\right]^{\mathrm{T}}$ is the velocity in $n_{s}$-frame with error $\delta \mathbf{V}^{n_{s}}=\left[\begin{array}{lll}\delta V_{\mathrm{E}} & \delta V_{\mathrm{N}} & \delta V_{\mathrm{U}}\end{array}\right]^{\mathrm{T}}$; $\mathbf{f}^{n_{s}}=\left[\begin{array}{lll}f_{\mathrm{E}} & f_{\mathrm{N}} & f_{\mathrm{U}}\end{array}\right]^{\mathrm{T}}$ is the specific force measured by the accelerometers of sub-IMU expressed in $n_{s}$-frame; $\boldsymbol{\omega}_{i e}^{n_{s}}$ is the rotation velocity of the $e$-frame relative to the $i$-frame expressed in $n_{s}$-frame with error $\delta \boldsymbol{\omega}_{i e}^{n_{s}} ; \boldsymbol{\omega}_{e n_{s}}^{n_{s}}$ is the rotation velocity of the $n_{s}$-frame relative to the $e$-frame expressed in $n_{s}$-frame with error $\delta \boldsymbol{\omega}_{e n_{s}}^{n_{s}} ; \nabla^{b_{s}}$ is the accelerometer random bias of slave system in $b_{s}$-frame, which consists of random constant bias $\nabla_{c}^{b_{s}}$ and Gaussian white noise $\omega_{\nabla}^{b_{s}}[28,29]$ with $\nabla_{c}^{b_{s}}=\left[\begin{array}{lll}\nabla_{x}^{b_{s}} & \nabla_{y}^{b_{s}} & \nabla_{z}^{b_{s}}\end{array}\right]^{\mathrm{T}}$ and $\boldsymbol{\omega}_{\nabla}^{b_{s}}=\left[\begin{array}{lll}\omega_{\nabla_{x}}^{b_{s}} & \omega_{\nabla_{y}}^{b_{s}} & \omega_{\nabla_{z}}^{b_{s}}\end{array}\right]^{\mathrm{T}}$; $R_{\mathrm{M}}$ and $R_{\mathrm{N}}$ denote the meridian and transverse radius of curvature, respectively; $L$ and $H$ denote the latitude and altitude, respectively. The symbols $\delta L, \delta \lambda$, and $\delta H$ denote the error of the latitude, longitude, and altitude, respectively. $\sec L=1 / \cos L$.

Secondly, the differential equation of rigid misalignment angle and flexure angle is shown in the following equations:

$$
\begin{gathered}
\dot{\rho}_{j}=0, \\
\ddot{\theta}_{j}+2 \beta_{j} \dot{\theta}_{j}+\beta_{j}^{2} \theta_{j}=\eta_{j}, \\
j=x, y, z,
\end{gathered}
$$

where the flexure angle $\theta_{j}$ is described by the second-order Markov process [30]; $\beta_{j}=2.146 / \tau_{j}$ and $\tau_{j}$ is the correlation time; $x, y$, and $z$ represent the axis of $b_{s}$-frame; $\eta_{j}$ is the Gaussian white noise with covariance $Q_{\eta_{j}}=4 \beta_{j}^{3} \sigma_{j}^{2}$, and $\sigma_{j}^{2}$ is the covariance of $\theta_{j} ; \mathbf{Q}_{\eta}=\left[\begin{array}{lll}Q_{\eta_{x}} & Q_{\eta_{y}} & Q_{\eta_{z}}\end{array}\right]^{\mathrm{T}}$.

2.1.2. System State Equation. The system state equation can be described as follows:

$$
\dot{X}_{I}=\mathbf{F}_{I} X_{I}+\mathbf{G}_{I} W_{I},
$$

where the subscript $I$ represents the deformation measuring algorithm based on IMU; $\mathbf{X}_{I}$ is a $24 \times 1$ error state vector defined by $\mathbf{X}_{I}=\left[\begin{array}{lllll}\phi_{\mathrm{E}} & \phi_{\mathrm{N}} & \phi_{\mathrm{U}} & \delta V_{\mathrm{E}} & \delta V_{\mathrm{N}}\end{array}\right.$

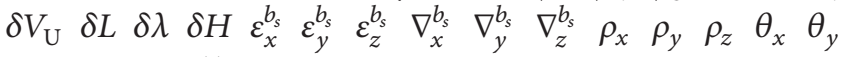
$\left.\begin{array}{llll}\theta_{z} & \dot{\theta}_{x} & \dot{\theta}_{y} & \dot{\theta}_{z}\end{array}\right]^{\mathrm{T}} ; \mathbf{F}_{I}$ and $\mathbf{G}_{I}$ are system transition matrix and system noise distribution matrix, respectively; the elements of $\mathbf{F}_{I}$ and $\mathbf{G}_{I}$ can be obtained from (2) and (3); system noise vector $W_{I}=\left[\begin{array}{lllllllll}\omega_{\varepsilon_{x}}^{b_{s}} & \omega_{\varepsilon_{y}}^{b_{s}} & \omega_{\varepsilon_{z}}^{b_{s}} & \omega_{\nabla_{x}}^{b_{s}} & \omega_{\nabla_{y}}^{b_{s}} & \omega_{\nabla_{z}}^{b_{s}} & \eta_{x} & \eta_{y} & \eta_{z}\end{array}\right]^{\mathrm{T}}$ is the zero-mean Gaussian white noise vector with covariance $\mathbf{Q}_{I}$ which consists of covariance $\mathbf{Q}_{\varepsilon}$ of gyro random drift, covariance $\mathbf{Q}_{\nabla}$ of accelerometer random bias, and $\mathbf{Q}_{\eta}$.

2.1.3. Measurement Equation. Based on the velocity plus attitude matching method, the measurement equation is given by

$$
\mathbf{Z}_{I}=\mathbf{H}_{I} \mathbf{X}_{I}+\mathbf{v}_{I}
$$

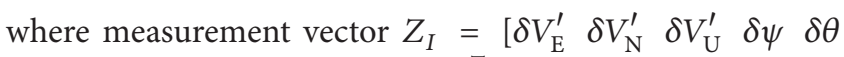
$\delta \gamma]^{\mathrm{T}} ; \delta \mathbf{V}^{\prime}=\left[\begin{array}{lll}\delta V_{\mathrm{E}}^{\prime} & \delta V_{\mathrm{N}}^{\prime} & \delta V_{\mathrm{U}}^{\prime}\end{array}\right]^{\mathrm{T}}$ denotes the differences of velocity between the slave system and master system after compensation for lever arm velocity; $\delta \psi, \delta \theta$, and $\delta \gamma$ denote the differences of heading, pitch, and roll between the slave system and master system, respectively; measurement noise $\mathbf{v}_{I}=\left[\begin{array}{llllll}v_{\delta V_{\mathrm{E}}} & v_{\delta V_{\mathrm{N}}} & v_{\delta V_{\mathrm{U}}} & v_{\delta \psi} & v_{\delta \theta} & v_{\delta \gamma}\end{array}\right]^{\mathrm{T}}$ is the zeromean Gaussian white noise sequence with covariance $\mathbf{R}_{I}$; measurement matrix $\mathbf{H}_{I}$ is given in (6); the details of $\mathbf{H}_{1}, \mathbf{H}_{2}$, 
and $\mathbf{H}_{3}$ can be found in $[31,32]$, and

$$
\mathbf{H}_{I}=\left[\begin{array}{cccccc}
\mathbf{0}_{3 \times 3} & \mathbf{I}_{3 \times 3} & \mathbf{0}_{3 \times 9} & \mathbf{0}_{3 \times 3} & \mathbf{0}_{3 \times 3} & \mathbf{0}_{3 \times 3} \\
\mathbf{H}_{1} & \mathbf{0}_{3 \times 3} & \mathbf{0}_{3 \times 9} & \mathbf{H}_{2} & \mathbf{H}_{3} & \mathbf{0}_{3 \times 3}
\end{array}\right] .
$$

$\delta \mathbf{V}^{\prime}$ is computed by

$$
\delta \mathbf{V}^{\prime}=\mathbf{V}^{n_{s}}-\mathbf{V}^{n_{m}}-\mathbf{V}_{r}^{n_{m}},
$$

where $\mathbf{V}^{n_{m}}$ is the velocity of the master system in $n_{m}$-frame and the lever arm velocity $\mathbf{V}_{r}^{n_{m}}$ can be calculated by [30]

$$
\mathbf{V}_{r}^{n_{m}}=\mathbf{C}_{b_{m}}^{n_{m}}\left(\boldsymbol{\omega}_{i b_{m}}^{b_{m}} \times \mathbf{r}^{b_{m}}\right)+\mathbf{C}_{b_{m}}^{n_{m}} \dot{\mathbf{r}}^{b_{m}}
$$

where $\mathbf{C}_{b_{m}}^{n_{m}}$ is the coordinate transformation matrix from $b_{m^{-}}$ frame to $n_{m}$-frame; $\boldsymbol{\omega}_{i b_{m}}^{b_{m}}$ is the rotation velocity of the $b_{m^{-}}$ frame relative to the $i$-frame expressed in the $b_{m}$-frame; $\mathbf{r}^{b_{m}}$ is the lever arm between the master system and slave system expressed in the $b_{m}$-frame.

\subsection{Mathematical Model of Deformation Measuring Algorithm} Based on Gs. This method needs three orthogonally mounted gyros as the slave system and main POS as the master system. The deformation is estimated by using the difference of gyros between the master system and slave system as the measurement of KF.

2.2.1. State Vector Selection. The rigid misalignment angle, flexure angle and its derivative, and gyro constant drifts of the master system and slave system are selected as the state vector:

$$
\begin{aligned}
& \mathbf{X}_{G}=\left[\rho_{x}, \rho_{y}, \rho_{z}, \theta_{x}, \theta_{y}, \theta_{z}, \dot{\theta}_{x}, \dot{\theta}_{y}, \dot{\theta}_{z}, \varepsilon_{x}^{b_{m}}, \varepsilon_{y}^{b_{m}}, \varepsilon_{z}^{b_{m}}, \varepsilon_{x}^{b_{s}}, \varepsilon_{y}^{b_{s}},\right. \\
&\left.\varepsilon_{z}^{b_{s}}\right]^{\mathrm{T}},
\end{aligned}
$$

where subscript $G$ represents the deformation measuring algorithm based on Gs; the symbols $\varepsilon_{j}^{b_{m}}$ and $\varepsilon_{j}^{b_{s}}(j=x, y, z)$ denote gyro random constant drift of the master system and slave system, respectively.

2.2.2. System State Equation. The system state equation is given by

$$
\dot{\mathbf{X}}_{G}=\mathbf{F}_{G} \mathbf{X}_{G}+\mathbf{G}_{G} \mathbf{W}_{G},
$$

where $\mathbf{F}_{G}$ and $\mathbf{G}_{G}$ are system transition matrix and system noise distribution matrix, respectively; system noise vector $\mathbf{W}_{G}=\left[\begin{array}{lll}\eta_{x} & \eta_{y} & \eta_{z}\end{array}\right]^{\mathrm{T}}$ is a zero-mean Gaussian white noise vector with covariance $\mathbf{Q}_{G}=\mathbf{Q}_{\eta}$.

The differential equation of rigid misalignment angle and flexure angle is the same as in (3). The differential equation of gyro random constant drift is given by

$$
\begin{aligned}
& \dot{\varepsilon}_{j}^{b_{m}}=0, \\
& \dot{\varepsilon}_{j}^{b_{s}}=0,
\end{aligned}
$$

According to (3) and (11), $\mathbf{F}_{G}$ and $\mathbf{G}_{G}$ are given as follows:

$$
\begin{gathered}
\mathbf{F}_{G}=\left[\begin{array}{ccccc}
\mathbf{0}_{3 \times 3} & \mathbf{0}_{3 \times 3} & \mathbf{0}_{3 \times 3} & \mathbf{0}_{3 \times 3} & \mathbf{0}_{3 \times 3} \\
\mathbf{0}_{3 \times 3} & \mathbf{0}_{3 \times 3} & \mathbf{I}_{3 \times 3} & \mathbf{0}_{3 \times 3} & \mathbf{0}_{3 \times 3} \\
\mathbf{0}_{3 \times 3} & \mathbf{B}_{1} & \mathbf{B}_{2} & \mathbf{0}_{3 \times 3} & \mathbf{0}_{3 \times 3} \\
\mathbf{0}_{3 \times 3} & \mathbf{0}_{3 \times 3} & \mathbf{0}_{3 \times 3} & \mathbf{0}_{3 \times 3} & \mathbf{0}_{3 \times 3} \\
\mathbf{0}_{3 \times 3} & \mathbf{0}_{3 \times 3} & \mathbf{0}_{3 \times 3} & \mathbf{0}_{3 \times 3} & \mathbf{0}_{3 \times 3}
\end{array}\right], \\
\mathbf{G}_{G}=\left[\begin{array}{lllll}
\mathbf{0}_{3 \times 3} & \mathbf{0}_{3 \times 3} & \mathbf{I}_{3 \times 3} & \mathbf{0}_{3 \times 3} & \mathbf{0}_{3 \times 3}
\end{array}\right]^{\mathrm{T}},
\end{gathered}
$$

where

$$
\begin{aligned}
\mathbf{B}_{1} & =\left[\begin{array}{ccc}
-\beta_{x}^{2} & 0 & 0 \\
0 & -\beta_{y}^{2} & 0 \\
0 & 0 & -\beta_{z}^{2}
\end{array}\right], \\
\mathbf{B}_{2} & =\left[\begin{array}{ccc}
-2 \beta_{x} & 0 & 0 \\
0 & -2 \beta_{y} & 0 \\
0 & 0 & -2 \beta_{z}
\end{array}\right] .
\end{aligned}
$$

2.2.3. Measurement Equation. The relationship between gyro outputs of the master system and slave system can be expressed by

$$
\boldsymbol{\Omega}^{b_{s}}+\boldsymbol{\varepsilon}^{b_{s}}=\mathbf{C}_{b_{m}}^{b_{s}}\left(\boldsymbol{\Omega}^{b_{m}}+\boldsymbol{\varepsilon}^{b_{m}}\right)+\dot{\boldsymbol{\theta}},
$$

where $\boldsymbol{\Omega}^{b_{m}}=\left[\begin{array}{lll}\omega_{x}^{b_{m}} & \omega_{y}^{b_{m}} & \omega_{z}^{b_{m}}\end{array}\right]^{\mathrm{T}}$ and $\boldsymbol{\Omega}^{b_{s}}=\left[\begin{array}{lll}\omega_{x}^{b_{s}} & \omega_{y}^{b_{s}} & \omega_{z}^{b_{s}}\end{array}\right]^{\mathrm{T}}$ are gyro angular velocity of the master system and slave system, respectively; $\dot{\boldsymbol{\theta}}=\left[\begin{array}{lll}\dot{\theta}_{x} & \dot{\theta}_{y} & \dot{\theta}_{z}\end{array}\right]^{\mathrm{T}} ; \boldsymbol{\varepsilon}^{b_{m}}$ is the gyro random drift of the master system in $b_{m}$-frame, which consists of random constant drift $\boldsymbol{\varepsilon}_{c}^{b_{m}}$ and Gaussian white noise $\boldsymbol{\omega}_{\varepsilon}^{b_{m}}$.

According to (1) and (14), the difference of gyro output between the master and slave systems is

$$
\begin{aligned}
\Delta \boldsymbol{\Omega} & =\boldsymbol{\Omega}^{b_{s}}-\boldsymbol{\Omega}^{b_{m}}=\mathbf{C}_{b_{m}}^{b_{s}} \boldsymbol{\Omega}^{b_{m}}+\mathbf{C}_{b_{m}}^{b_{s}} \boldsymbol{\varepsilon}^{b_{m}}+\dot{\boldsymbol{\theta}}-\boldsymbol{\varepsilon}^{b_{s}}-\boldsymbol{\Omega}^{b_{m}} \\
& =\left(\mathbf{C}_{b_{m}}^{b_{s}}-\mathbf{I}\right) \boldsymbol{\Omega}^{b_{s}}+\dot{\boldsymbol{\theta}}+\mathbf{C}_{b_{m}}^{b_{s}} \boldsymbol{\varepsilon}^{b_{m}}-\boldsymbol{\varepsilon}_{c}^{b_{s}} \\
& =[-(\boldsymbol{\rho}+\boldsymbol{\theta}) \times] \boldsymbol{\Omega}^{b_{s}}+\dot{\boldsymbol{\theta}}+\mathbf{C}_{b_{m}}^{b_{s}} \boldsymbol{\varepsilon}^{b_{m}}-\boldsymbol{\varepsilon}_{c}^{b_{s}} \\
& =\boldsymbol{\Omega}^{b_{s}} \times(\boldsymbol{\rho}+\boldsymbol{\theta})+\dot{\boldsymbol{\theta}}+\mathbf{C}_{b_{m}}^{b_{s}} \boldsymbol{\varepsilon}^{b_{m}}-\boldsymbol{\varepsilon}_{c}^{b_{s}} \\
& =\widehat{\boldsymbol{\Omega}}(\boldsymbol{\rho}+\boldsymbol{\theta})+\dot{\boldsymbol{\theta}}+\mathbf{C}_{b_{m}}^{b_{s}} \boldsymbol{\varepsilon}^{b_{m}}-\boldsymbol{\varepsilon}_{c}^{b_{s}} \\
& \approx \widehat{\boldsymbol{\Omega}}(\boldsymbol{\rho}+\boldsymbol{\theta})+\dot{\boldsymbol{\theta}}+\boldsymbol{\varepsilon}^{b_{m}}-\boldsymbol{\varepsilon}^{b_{s}},
\end{aligned}
$$

where

$$
\widehat{\boldsymbol{\Omega}}=\left[\begin{array}{ccc}
0 & -\omega_{z}^{b_{m}} & \omega_{y}^{b_{m}} \\
\omega_{z}^{b_{m}} & 0 & -\omega_{x}^{b_{m}} \\
-\omega_{y}^{b_{m}} & \omega_{x}^{b_{m}} & 0
\end{array}\right] .
$$
yields

Substituting $\boldsymbol{\varepsilon}^{b_{m}}=\boldsymbol{\varepsilon}_{c}^{b_{m}}+\boldsymbol{\omega}_{\varepsilon}^{b_{m}}$ and $\boldsymbol{\varepsilon}^{b_{s}}=\boldsymbol{\varepsilon}_{c}^{b_{s}}+\boldsymbol{\omega}_{\varepsilon}^{b_{s}}$ into (15) $j=x, y, z$.

$$
\Delta \boldsymbol{\Omega}=\widehat{\boldsymbol{\Omega}}(\boldsymbol{\rho}+\boldsymbol{\theta})+\dot{\boldsymbol{\theta}}+\boldsymbol{\varepsilon}_{c}^{b_{m}}-\boldsymbol{\varepsilon}_{c}^{b_{s}}+\boldsymbol{\omega}_{\varepsilon}^{b_{m}}-\boldsymbol{\omega}_{\varepsilon}^{b_{s}} .
$$


Based on (17), the measurement equation is given as follows:

$$
\mathbf{Z}_{G}=\mathbf{H}_{G} \mathbf{X}_{G}+\mathbf{v}_{G}
$$

where measurement vector $\mathbf{Z}_{G}=\Delta \boldsymbol{\Omega}$; measurement matrix $\mathbf{H}_{G}=\left[\begin{array}{lllll}\widehat{\boldsymbol{\Omega}} & \widehat{\boldsymbol{\Omega}} & \mathbf{I}_{3 \times 3} & \mathbf{I}_{3 \times 3} & -\mathbf{I}_{3 \times 3}\end{array}\right] ;$ measurement noise $\mathbf{v}_{G}=$ $\left[\begin{array}{lll}v_{\delta \omega_{x}} & v_{\delta \omega_{y}} & v_{\delta \omega_{z}}\end{array}\right]^{\mathrm{T}}$ is a zero-mean Gaussian white noise sequence with covariance $\mathbf{R}_{G}$.

2.3. Mathematical Modeling of Deformation Measuring Algorithm Based on As. In this method, taking three orthogonally mounted accelerometers as the slave system and main POS as the master system, the difference of accelerometers between the master system and slave system is selected as the measurement of KF to estimate the deformation.

\subsubsection{State Vector Selection. The state vector is defined by}

$$
\begin{gathered}
\mathbf{X}_{A}=\left[\rho_{x}, \rho_{y}, \rho_{z}, \theta_{x}, \theta_{y}, \theta_{z}, \dot{\theta}_{x}, \dot{\theta}_{y}, \dot{\theta}_{z}, \nabla_{x}^{b_{m}}, \nabla_{y}^{b_{m}}, \nabla_{z}^{b_{m}}, \nabla_{x}^{b_{s}}, \nabla_{y}^{b_{s}},\right. \\
\left.\nabla_{z}^{b_{s}}\right]^{\mathrm{T}},
\end{gathered}
$$

where subscript $A$ represents the deformation measuring algorithm based on As; $\nabla_{j}^{b_{m}}$ and $\nabla_{j}^{b_{s}}(j=x, y, z)$ are accelerometer random constant bias of the master system and slave system, respectively.

2.3.2. System State Equation. The system state equation is given by

$$
\dot{\mathbf{X}}_{A}=\mathbf{F}_{A} \mathbf{X}_{A}+\mathbf{G}_{A} \mathbf{W}_{A}
$$

where $\mathbf{F}_{A}$ and $\mathbf{G}_{A}$ are system transition matrix and system noise distribution matrix, respectively; system noise vector $\mathbf{W}_{A}=\left[\begin{array}{lll}\eta_{x} & \eta_{y} & \eta_{z}\end{array}\right]^{\mathrm{T}}$ is a zero-mean Gaussian white noise vector with covariance $\mathbf{Q}_{A}=\mathbf{Q}_{\eta}$.

The differential equation of rigid misalignment angle and flexure angle is the same as in (3). The differential equation of accelerometer random constant bias is given by

$$
\begin{aligned}
& \dot{\nabla}_{j}^{b_{m}}=0, \\
& \dot{\nabla}_{j}^{b_{s}}=0, \\
& \quad j=x, y, z .
\end{aligned}
$$

The expression of $\mathbf{F}_{A}$ and $\mathbf{G}_{A}$ can be obtained from (3) and (21). Furthermore, $\mathbf{F}_{A}=\mathbf{F}_{G}$ and $\mathbf{G}_{A}=\mathbf{G}_{G}$.

2.3.3. Measurement Equation. The relationship between the accelerometer outputs of the master system and slave system can be expressed by

$$
\mathbf{f}^{b_{s}}+\nabla^{b_{s}}=\mathbf{C}_{b_{m}}^{b_{s}}\left(\mathbf{f}^{b_{m}}+\nabla^{b_{m}}+\mathbf{a}_{r}^{b_{m}}\right)
$$

where $\mathbf{f}^{b_{m}}=\left[\begin{array}{lll}f_{x}^{b_{m}} & f_{y}^{b_{m}} & f_{z}^{b_{m}}\end{array}\right]^{\mathrm{T}}$ and $\mathbf{f}^{b_{s}}=\left[\begin{array}{lll}f_{x}^{b_{s}} & f_{y}^{b_{s}} & f_{z}^{b_{s}}\end{array}\right]^{\mathrm{T}}$ are the specific forces measured by the accelerometers of the master system and slave system, respectively; $\nabla^{b_{m}}$ is the accelerometer random bias of the master system in $b_{m}$-frame, which consists of random constant bias $\nabla_{c}^{b_{m}}$ and Gaussian white noise $\boldsymbol{\omega}_{\nabla}^{b_{m}} ; \mathbf{a}_{r}^{b_{m}}$ is the relative acceleration between the master system and slave system caused by lever arm; $\mathbf{a}_{r}^{b_{m}}$ is given by

$$
\begin{aligned}
\mathbf{a}_{r}^{b_{m}}= & \boldsymbol{\Omega}^{b_{m}} \times \boldsymbol{\Omega}^{b_{m}} \times \mathbf{r}^{b_{m}}+\dot{\boldsymbol{\Omega}}^{b_{m}} \times \mathbf{r}^{b_{m}}+2\left(\boldsymbol{\Omega}^{b_{m}} \times \dot{\mathbf{r}}^{b_{m}}\right) \\
& +\ddot{\mathbf{r}}^{b_{m}} .
\end{aligned}
$$

According to (1) and (22), the difference of accelerometer output between the master system and slave system is

$$
\begin{aligned}
\Delta \mathbf{f} & =\left(\mathbf{f}^{b_{s}}-\mathbf{C}_{b_{m}}^{b_{s}} \mathbf{a}_{r}^{b_{m}}\right)-\mathbf{f}^{b_{m}} \\
& =\mathbf{C}_{b_{m}}^{b_{s}} \mathbf{f}^{b_{m}}+\mathbf{C}_{b_{m}}^{b_{s}} \nabla^{b_{m}}-\nabla^{b_{s}}-\mathbf{f}^{b_{m}} \\
& =\left(\mathbf{C}_{b_{m}}^{b_{s}}-\mathbf{I}\right) \mathbf{f}^{b_{m}}+\mathbf{C}_{b_{m}}^{b_{s}} \nabla^{b_{m}}-\nabla^{b_{s}} \\
& =[-(\boldsymbol{\rho}+\boldsymbol{\theta}) \times] \mathbf{f}^{b_{m}}+\mathbf{C}_{b_{m}}^{b_{s}} \nabla^{b_{m}}-\nabla^{b_{s}} \\
& =\mathbf{f}^{b_{m}} \times(\boldsymbol{\rho}+\boldsymbol{\theta})+\mathbf{C}_{b_{m}}^{b_{s}} \nabla^{b_{m}}-\nabla^{b_{s}} \\
& =\widehat{\mathbf{f}}(\boldsymbol{\rho}+\boldsymbol{\theta})+\mathbf{C}_{b_{m}}^{b_{s}} \nabla^{b_{m}}-\nabla^{b_{s}} \\
& \approx \widehat{\mathbf{f}}(\boldsymbol{\rho}+\boldsymbol{\theta})+\nabla^{b_{m}}-\nabla^{b_{s}},
\end{aligned}
$$

where

$$
\widehat{\mathbf{f}}=\left[\begin{array}{ccc}
0 & -f_{z}^{b_{m}} & f_{y}^{b_{m}} \\
f_{z}^{b_{m}} & 0 & -f_{x}^{b_{m}} \\
-f_{y}^{b_{m}} & f_{x}^{b_{m}} & 0
\end{array}\right] .
$$
yields

Substituting $\nabla^{b_{m}}=\nabla_{c}^{b_{m}}+\boldsymbol{\omega}_{\nabla}^{b_{m}}$ and $\nabla^{b_{s}}=\nabla_{c}^{b_{s}}+\boldsymbol{\omega}_{\nabla}^{b_{s}}$ into (24)

$$
\Delta \mathbf{f}=\widehat{\mathbf{f}}(\boldsymbol{\rho}+\boldsymbol{\theta})+\nabla_{c}^{b_{m}}-\nabla_{c}^{b_{s}}+\boldsymbol{\omega}_{\nabla}^{b_{m}}-\boldsymbol{\omega}_{\nabla}^{b_{s}} .
$$

Based on (26), the measurement equation is

$$
\mathbf{Z}_{A}=\mathbf{H}_{A} \mathbf{X}_{A}+\mathbf{v}_{A},
$$

where measurement vector $\mathbf{Z}_{A}=\Delta \mathbf{f}$; measurement matrix $\mathbf{H}_{A}=\left[\begin{array}{lllll}\widehat{\mathbf{f}} & \widehat{\mathbf{f}} & \mathbf{0}_{3 \times 3} & \mathbf{I}_{3 \times 3} & -\mathbf{I}_{3 \times 3}\end{array}\right]$; measurement noise $\mathbf{v}_{A}=$ $\left[\begin{array}{lll}v_{\delta f_{x}} & v_{\delta f_{y}} & v_{\delta f_{z}}\end{array}\right]^{\mathrm{T}}$ is a zero-mean Gaussian white noise sequence with covariance $\mathbf{R}_{A}$.

\section{Simulation and Semiphysical Simulation}

In order to verify the estimation effect of methods based on Gs and As, respectively, proposed in Section 2, a long rod with a master system and a slave system installed on both ends is a better way. But in this case, it is not possible to know the exact value of deformation between the master system and slave system. Therefore, flight simulation and vehicle semiphysical simulation are provided and compared with the method based on IMU in this section. 
TABLE 1: Parameters setting of simulation trajectory.

\begin{tabular}{lc}
\hline Time $(\mathrm{s})$ & Motion state \\
\hline $0-100$ & Uniform linear motion \\
$100-200$ & Turn $60^{\circ}$ clockwise \\
$200-300$ & Turn $60^{\circ}$ anticlockwise \\
$300-700$ & Uniform linear motion \\
$700-900$ & Turn $180^{\circ}$ clockwise \\
$900-1300$ & Uniform linear motion \\
\hline
\end{tabular}

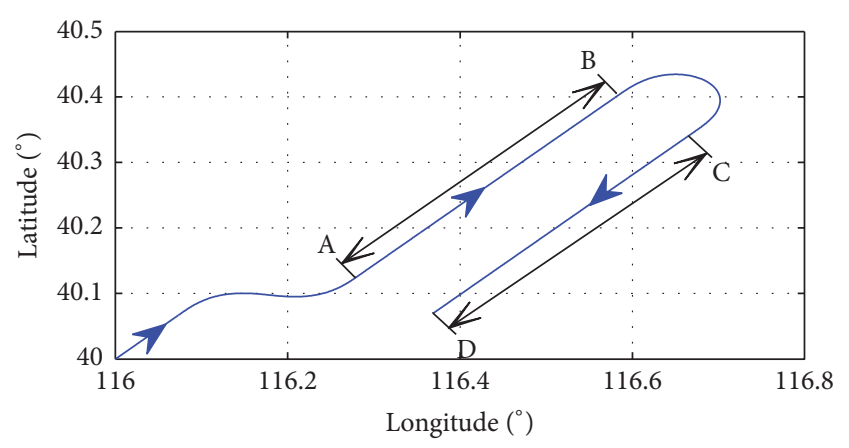

FIGURE 2: Plane trajectory with S-shaped maneuver and U-shaped flight.

3.1. Flight Simulation and Analysis. From the attitude transfer relationship shown in (1), it can be seen that the attitude difference between the master system and slave system, also called the system error angle, is determined by the sum of rigid misalignment angle and flexure angle. Therefore, not only should the estimation accuracy of the flexure angle or the rigid misalignment angle be evaluated, but also the estimation accuracy of the system angle error should be evaluated. In connection with this, the estimation error curves of flexure angle, rigid misalignment angle, and system error angle are given in the simulation, and the estimation error of the system error angle is used to evaluate the measuring precision of each method.

3.1.1. Design of Simulation. In this paper, the typical " $\mathrm{S}+\mathrm{U}$ "shaped trajectory of airborne earth observation is simulated. The plane trajectory and trajectory parameters are shown in Figure 2 and Table 1, respectively. Total flight time is $1300 \mathrm{~s}$. $\mathrm{AB}$ and $\mathrm{CD}$ section can be regarded as the imaging section in Figure 2. Initial heading angle, pitch angle, and roll angle are $40^{\circ}, 0^{\circ}$, and $0^{\circ}$, respectively. The flight velocity is $100 \mathrm{~m} / \mathrm{s}$ and the altitude is $500 \mathrm{~m}$. This simulation has been performed ten times.

The measurement noise of the main POS (as the master system) at heading, pitch, roll, and velocity are $0.02^{\circ}(1 \sigma)$, $0.005^{\circ}(1 \sigma), 0.005^{\circ}(1 \sigma)$, and $0.03 \mathrm{~m} / \mathrm{s}(1 \sigma)$, respectively. Both gyro constant drift and random drift of the main POS are $0.01^{\circ} / \mathrm{h}$. Both accelerometer constant bias and random bias of the main POS and slave system are $50 \mu \mathrm{g}$. Both gyro constant and random drift of the slave system are $0.1^{\circ} / \mathrm{h}$. The misalignment angle of the slave system relative to the main POS is given as $\boldsymbol{\rho}=\left[\begin{array}{lll}0.5^{\circ} & 0.5^{\circ} & 0.5^{\circ}\end{array}\right]^{\mathrm{T}}$, and the lever arm between the main POS and slave system is $\mathbf{r}^{b_{m}}=$ $\left[\begin{array}{llll}5 \mathrm{~m} & 0.1 \mathrm{~m} & 0.1 \mathrm{~m}\end{array}\right]^{\mathrm{T}}$. The data update rate of the main system and slave system is $100 \mathrm{~Hz}$. For the deformation measurement of the wing, the flexure angle rotated around the vertical axis is big, while the flexure angles around the other two axes are small. Accordingly, the correlation times of the second-order Markov processes are selected as 2, 5, and 2, and the covariances of flexure angle are $0.01,0.15$, and 0.01 , respectively. The curves of flexure angle and flexure angle rate are shown in Figures 3 and 4.

Data Generation. A trajectory generator is used to generate the theoretical data of the scheduled flight trajectory, which include position, velocity, attitude, and the output data of gyros and accelerometers. The real outputs of the main POS are obtained by adding the corresponding measurement noise to the theoretical position, velocity, and attitude. Then, the theoretical outputs of gyros and accelerometers are converted by rigid misalignment angles and flexure angles, and the constant noise and random noise are added to be the inertial sensor outputs of the slave system.

3.1.2. Simulation Results Analysis. Figures 5-7 show the estimate error curves of deformation measuring method based on IMU, Gs, and As, respectively, including the estimate errors of rigid misalignment angle, flexure angle, and system error angle. For improving the estimate accuracy, a maneuver is added in the S-shape of the flight trajectory and the system error angle estimate errors are shown in Figure 8. The details of the maneuver are as follows: the flight velocity of S-shape is increased equably from $100 \mathrm{~m} / \mathrm{s}$ to $300 \mathrm{~m} / \mathrm{s}$ between $100 \mathrm{~s}$ and $200 \mathrm{~s}$ and then decreased equably to $100 \mathrm{~m} / \mathrm{s}$ between $200 \mathrm{~s}$ and $300 \mathrm{~s}$. This kind of maneuver is very easy to implement for earth observation aircraft, because it is usually necessary to make $S$ or other types of maneuver to improve the estimation precision of POS before the aircraft enters the observation area.

Besides, the Root Mean Square Error (RMSE) and Standard Deviation (STD) values of system error angle estimate errors in imaging segments $\mathrm{AB}$ and $\mathrm{CD}$ are counted and shown in Table 2, where all values are the average of ten simulations. Since the maneuver mentioned above only affects the method based on Gs (it can be seen from Table 2), only the system error angle estimate errors of method based on IMU, Gs without this maneuver, and method based on Gs with this maneuver are shown in Figure 8.

Figures 5 and 6 show that the estimation accuracies of flexure angle and rigid misalignment angle are not good at the same time and coupled with each other, while the sum of two estimation errors of system error angle shown in Figure 7 is relatively stable on each axis.

Figures 7 and 8 and Table 2 show that the deformation measurement based on IMU has the highest estimation accuracy without any additional maneuver, followed by the method based on Gs, and the method based on As is the worst. The velocity maneuvers in S-shape can improve the estimation effect of the method based on Gs, especially 
TABLE 2: Estimate errors of system error angle in simulation test $\left(^{\prime}\right)$.

\begin{tabular}{|c|c|c|c|c|c|c|}
\hline \multirow{2}{*}{ Errors } & \multicolumn{3}{|c|}{ No variation at velocity in S-shape } & \multicolumn{3}{|c|}{ Variation at velocity in S-shape } \\
\hline & IMU & Gs & As & IMU & Gs & As \\
\hline \multicolumn{7}{|l|}{ STD } \\
\hline$x$-axis & 0.4108 & 0.4593 & 17.0632 & 0.4086 & 0.4333 & 17.0642 \\
\hline$y$-axis & 0.3832 & 0.2834 & 2.6423 & 0.3882 & 0.2403 & 2.6825 \\
\hline$z$-axis & 1.1755 & 0.4152 & 435.9763 & 1.0088 & 0.3902 & 437.4117 \\
\hline \multicolumn{7}{|l|}{ RMSE } \\
\hline$x$-axis & 0.6643 & 1.7323 & 24.2849 & 0.6546 & 0.8323 & 24.3184 \\
\hline$y$-axis & 0.5720 & 1.3091 & 3.9038 & 0.5943 & 0.6703 & 3.9360 \\
\hline$z$-axis & 2.1673 & 7.7563 & 1152.3785 & 2.1943 & 2.2219 & 1761.8890 \\
\hline
\end{tabular}
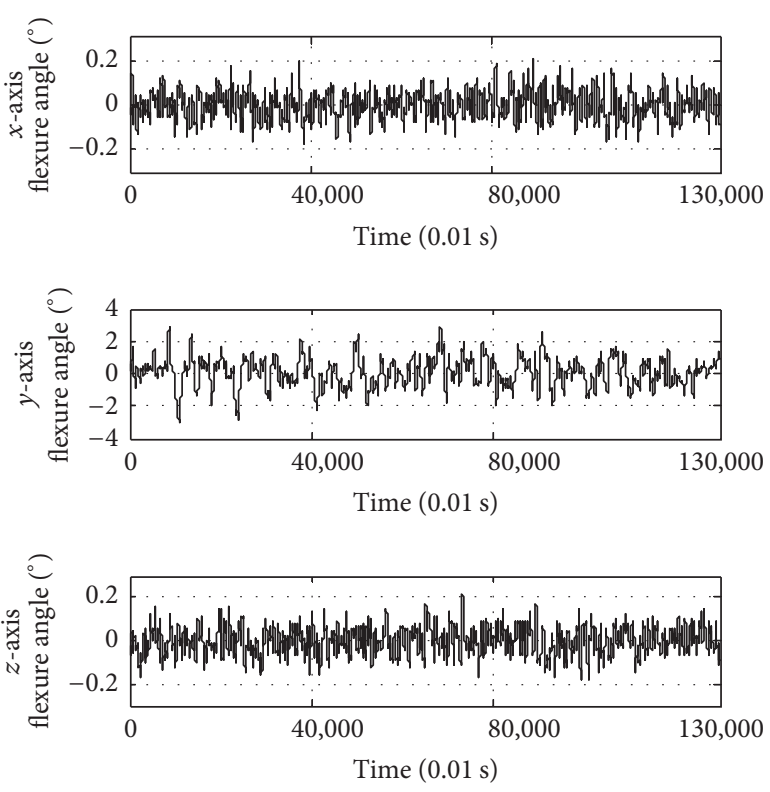

FIgURE 3: Flexure angle.

the RMSE, and obtain the estimation precision close to the method based on IMU. It is worth mentioning that although the method based on As has very poor estimation accuracy on $x$-axis and $z$-axis, the STD and RMES of system error angle estimation error on $y$-axis are only $3.3^{\prime}$ and $5^{\prime}$, respectively, which is very small relative to the flexure angle on $y$-axis shown in Figure 3.

3.2. Semiphysical Simulation and Analysis. A real road experiment is carried out in Shahe Town, Changping District, Beijing, China. In this vehicle experiment, a high precision POS is used as the main system, whose gyros, accelerometers, position, velocity, and attitude output are recorded. Based on these data, the flexure and misalignment sets in Section 3 are added, and then the theoretical data of the slave system can be obtained. After considering the error of the gyro and accelerometer in the slave system, the real output of the gyros and accelerometers in the slave system can be simulated.
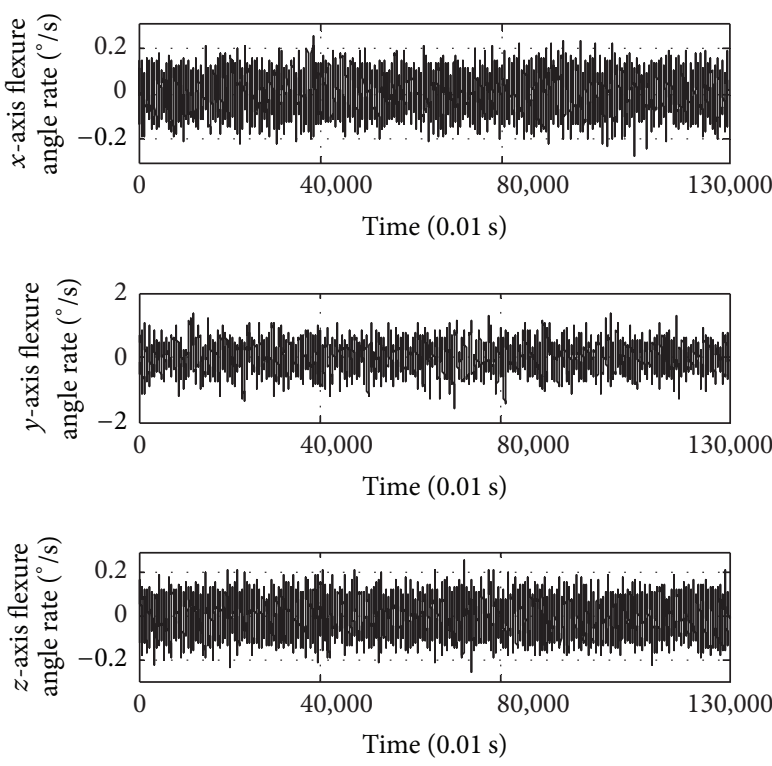

FIGURE 4: Flexure angle rate.

3.2.1. Hardware Configuration. The van and the sensors installation are shown in Figures 9 and 10, respectively. The high-precision POS, developed by the Integrated System Research Group at Beihang University, Beijing, China, consists of a laser gyro-based IMU, a PCS, and Novatel DLV3 GPS receiver (based on Novatel OEMV-3 receiver board) and has mobile station and base station equipment with $20 \mathrm{~Hz}$ output rate [33]. The trajectory of the test is shown in Figure 11 and the total test time is $1500 \mathrm{~s}$. Figure 12 shows the van's velocity and acceleration.

The specifications of the high-precision POS inertial sensors applied in the laser gyro-based IMU and position, velocity, and attitude postprocessing output are listed in Table 3. The inertial sensors' errors of the simulated slave system are the same as these used in Section 3. The output rate of the main POS is $100 \mathrm{~Hz}$.

3.2.2. Semiphysical Simulation Results Analysis. Figure 13 shows the system error angle estimate error curves of deformation measuring method based on IMU, Gs, and As. In 

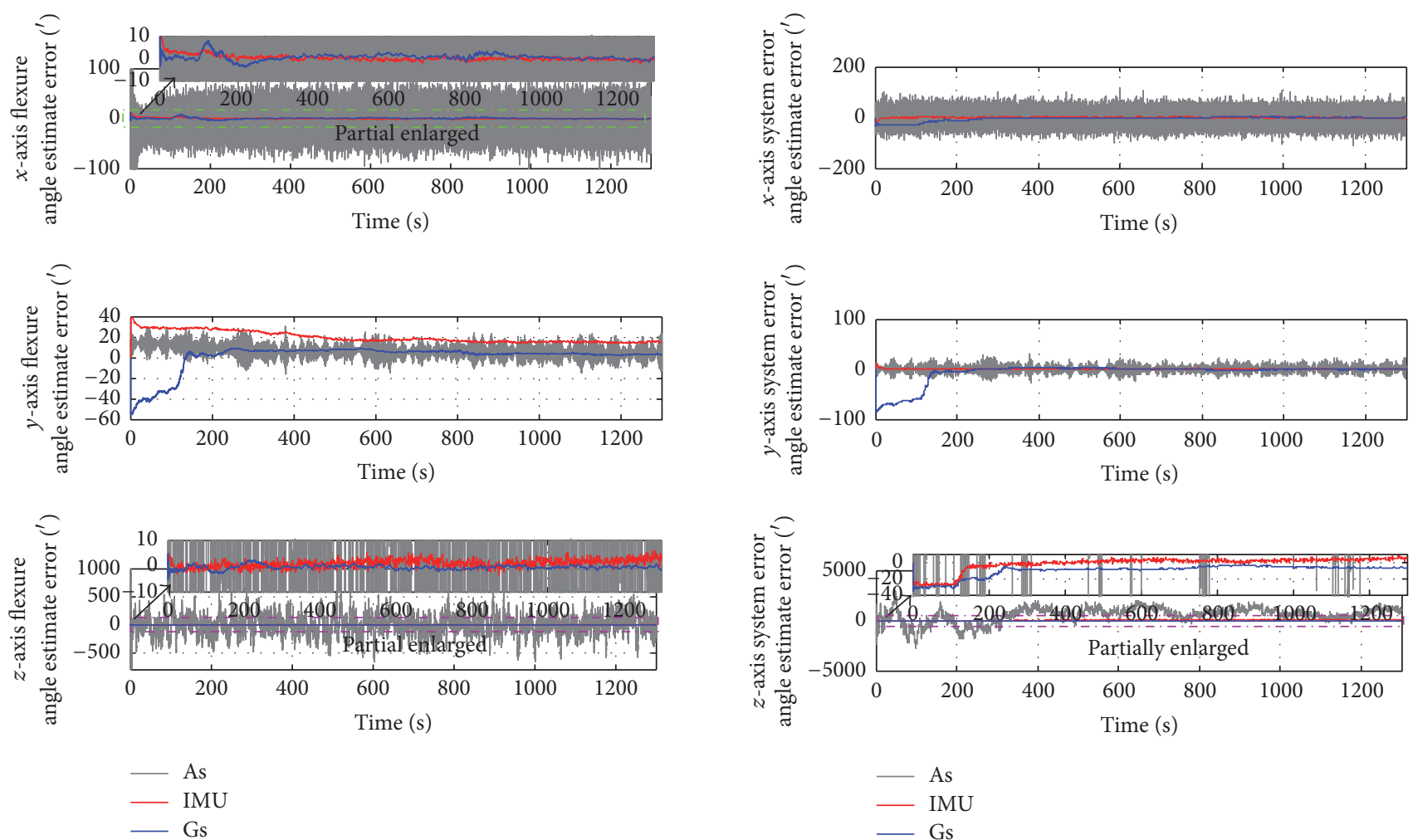

FIGURE 5: Flexure angle estimate error in simulation test.
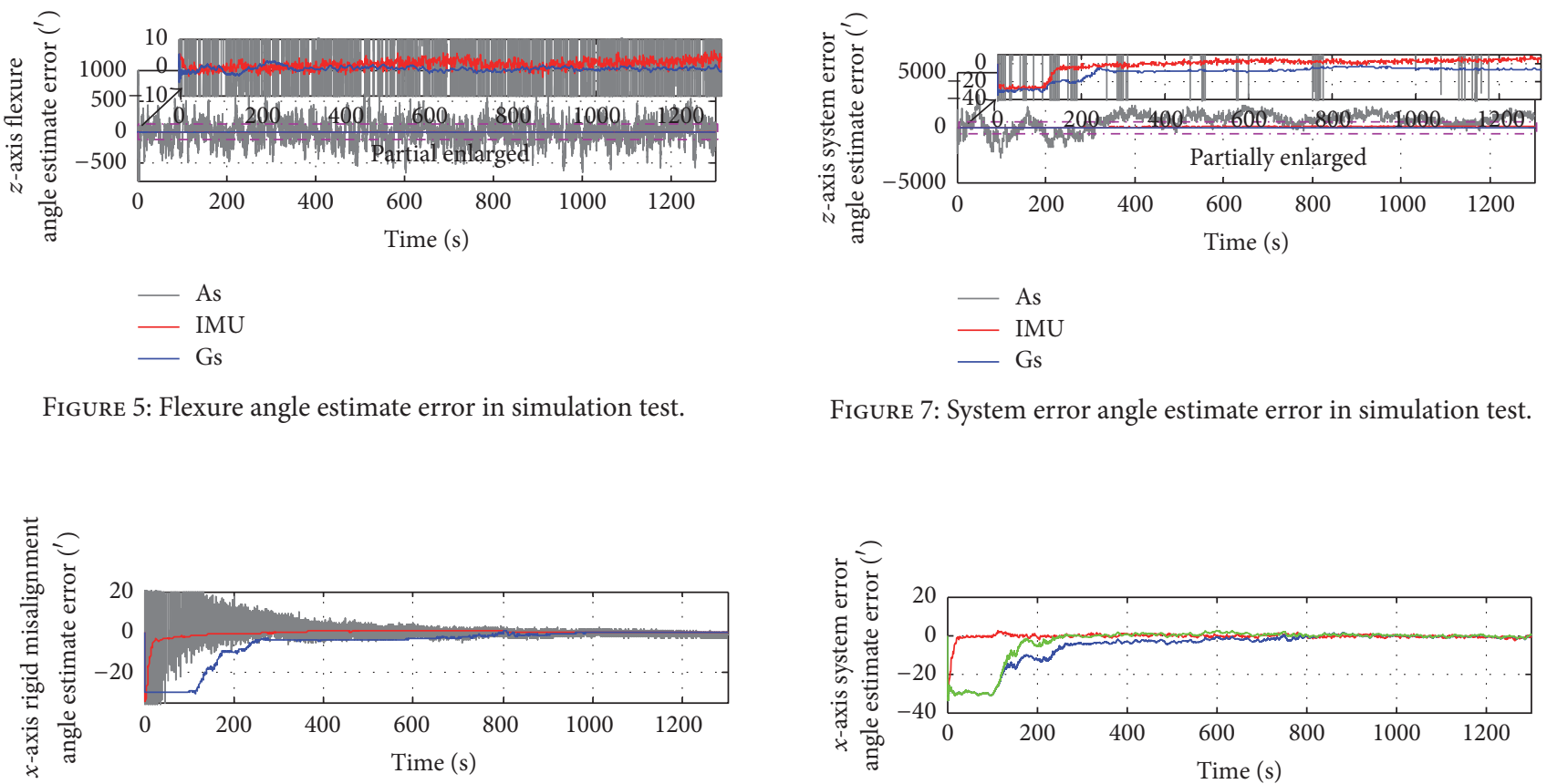

FIGURE 7: System error angle estimate error in simulation test.
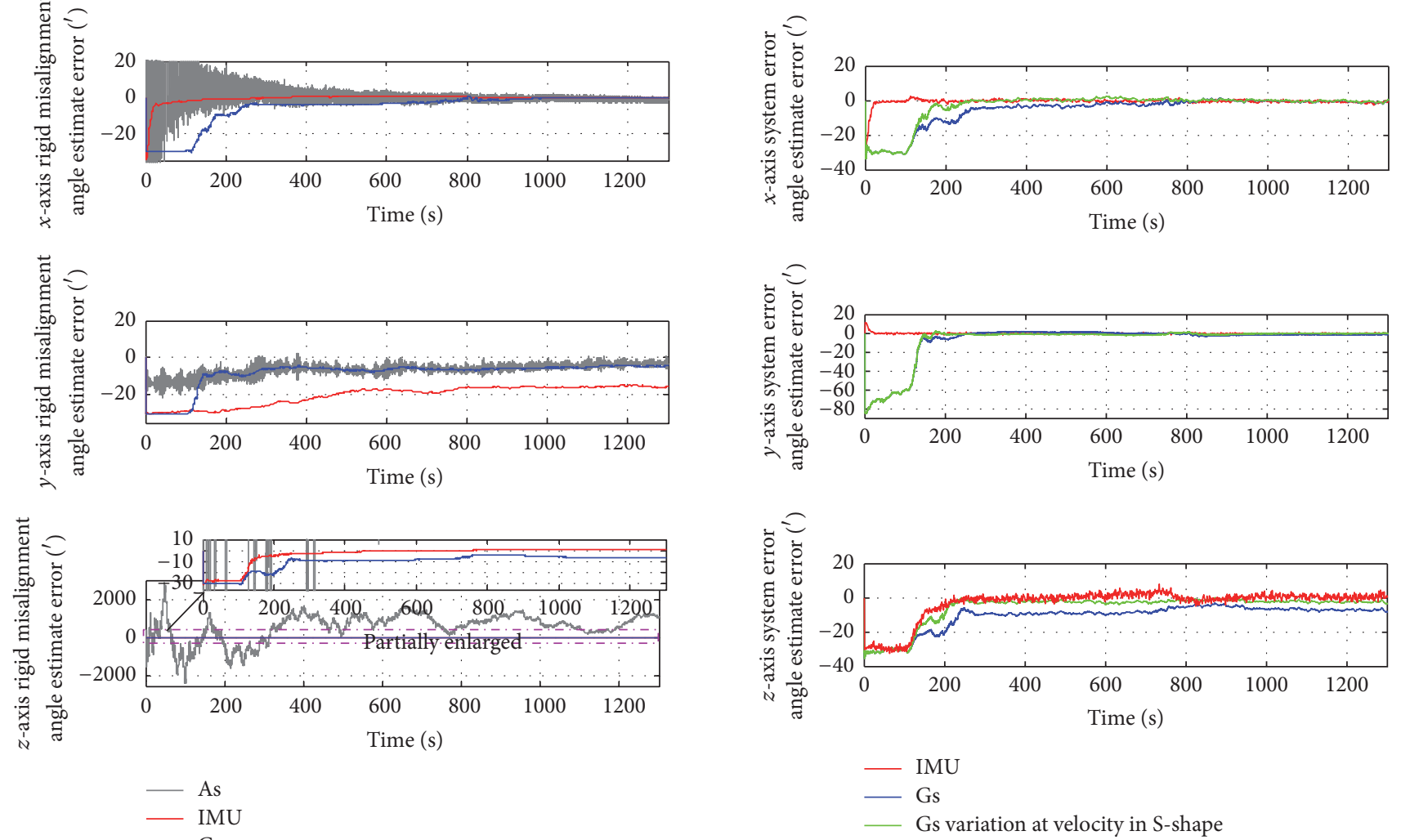

FIGURE 6: Rigid misalignment angle estimate error in simulation test.

FIGURE 8: System error angle estimate error of method based on IMU and Gs in simulation test. 

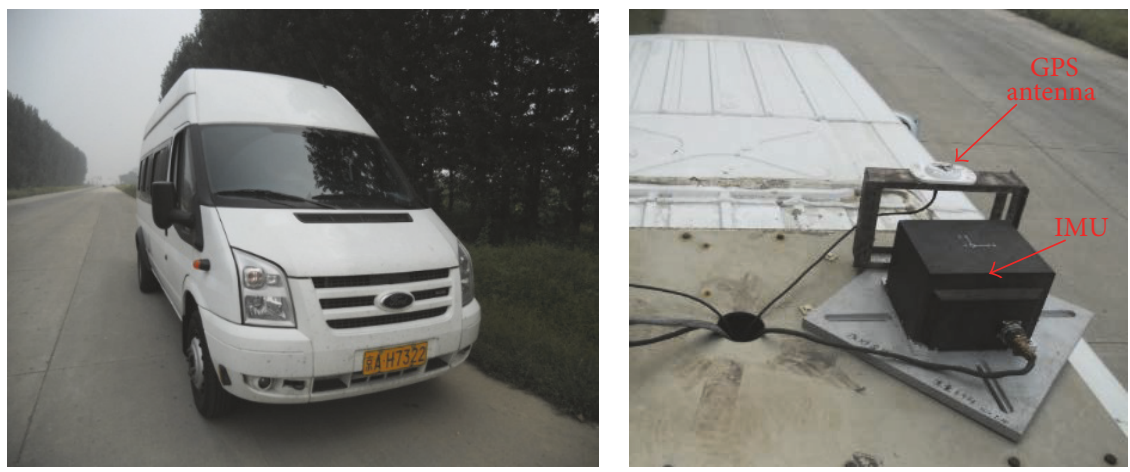

FIGURE 9: The van and sensors installed on the top of the van.
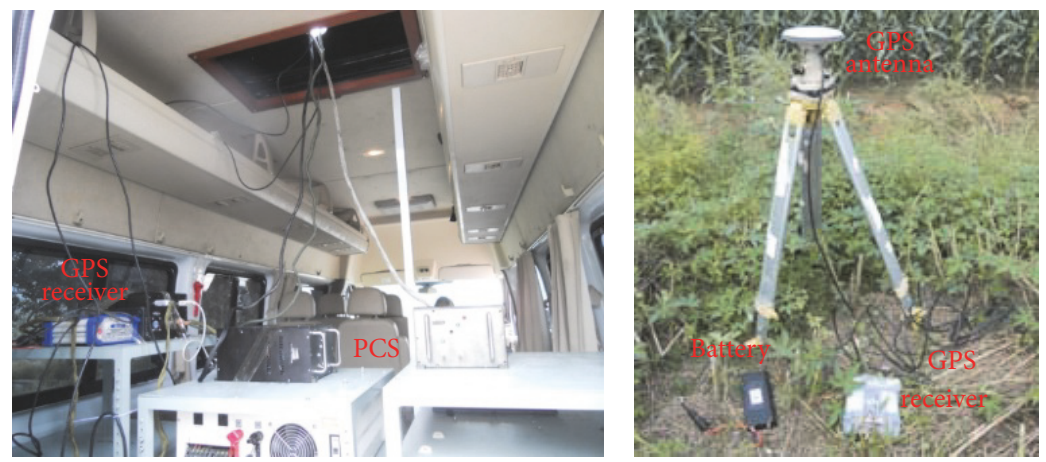

FIGURE 10: Sensors installed in the van and the base station equipment of GPS.

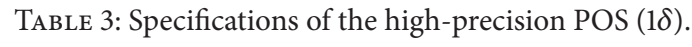

\begin{tabular}{lc}
\hline Parameters & Values \\
\hline Laser gyro & \\
$\quad$ Constant drift & $0.01^{\circ} / \mathrm{h}$ \\
Random drift & $0.01^{\circ} / \mathrm{h}$ \\
Accelerometer & \\
$\quad$ Constant bias & $50 \mu \mathrm{g}$ \\
$\quad$ Random bias & $50 \mu \mathrm{g}$ \\
Position & \\
Horizontal & $<0.1 \mathrm{~m}$ \\
$\quad$ Vertical & $<0.2 \mathrm{~m}$ \\
Velocity & $0.005 \mathrm{~m} / \mathrm{s}$ \\
Heading & $0.005^{\circ}$ \\
Pitch and roll & $0.0025^{\circ}$ \\
\hline
\end{tabular}

order to see the changing trends of errors more clearly, the error curves of the first two methods are shown in Figure 14, where L1 and L2 are two long straight segments in the trajectory. The statistics of system error angle estimate errors of L1 and L2 are given in Table 4.

Figures 13 and 14 and Table 4 show that the estimation accuracy of the method based on Gs is close to that of IMU method. In the horizontal direction, the estimation accuracy of the IMU method is more stable and the Gs method is greatly influenced by the turning of the vehicle; in the vertical direction, since there is not any maneuver and the vehicle began to move in a straight line, the estimation accuracy of the IMU method is gradually improved with the turn maneuver, and filtering is also gradually stabilized, while the Gs method can converge quickly. The method based on As has the largest estimation error, but its STD and RMES of system error angle estimation error on $y$-axis are no more than $30^{\prime}$. Overall, the vehicle experiment results and the simulation results are basically similar and consistent.

\section{Conclusion}

In this paper, the mathematical models of deformation measurement based on Gs and As are derived, respectively, and compared with the deformation measuring method based on IMU. The results of simulation and semiphysical simulation show that the measuring method based on Gs can achieve similar estimation accuracy to the method based on IMU. Since the method based on IMU has the disadvantages of large size, high cost, and heavy weight, when the accuracy requirement is not very high and can be met by the method based on Gs or As, the measurement equipment will be greatly simplified. In particular, the method based on As has a good estimation accuracy on $y$-axis, which is a better choice for the cases with large deformation only on $y$-axis and strict 


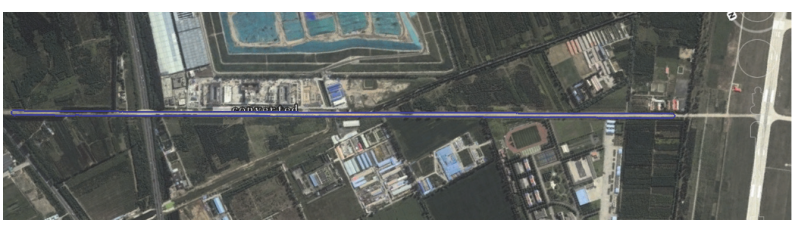

FIGURE 11: Trajectory of the experiment.
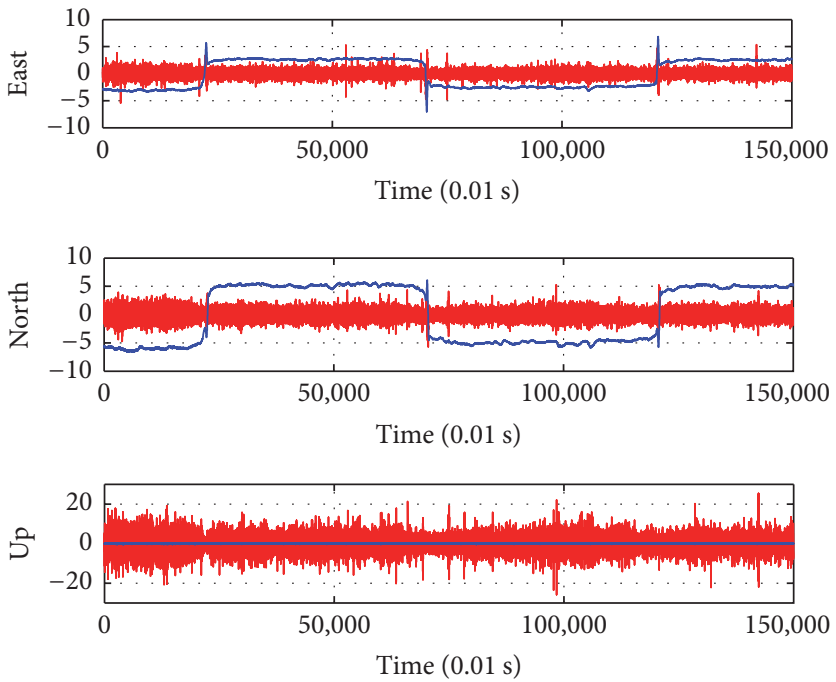

- Acceleration $\left(\mathrm{m} / \mathrm{s}^{2}\right)$

— Velocity $(\mathrm{m} / \mathrm{s})$

Figure 12: The van's velocity and acceleration.
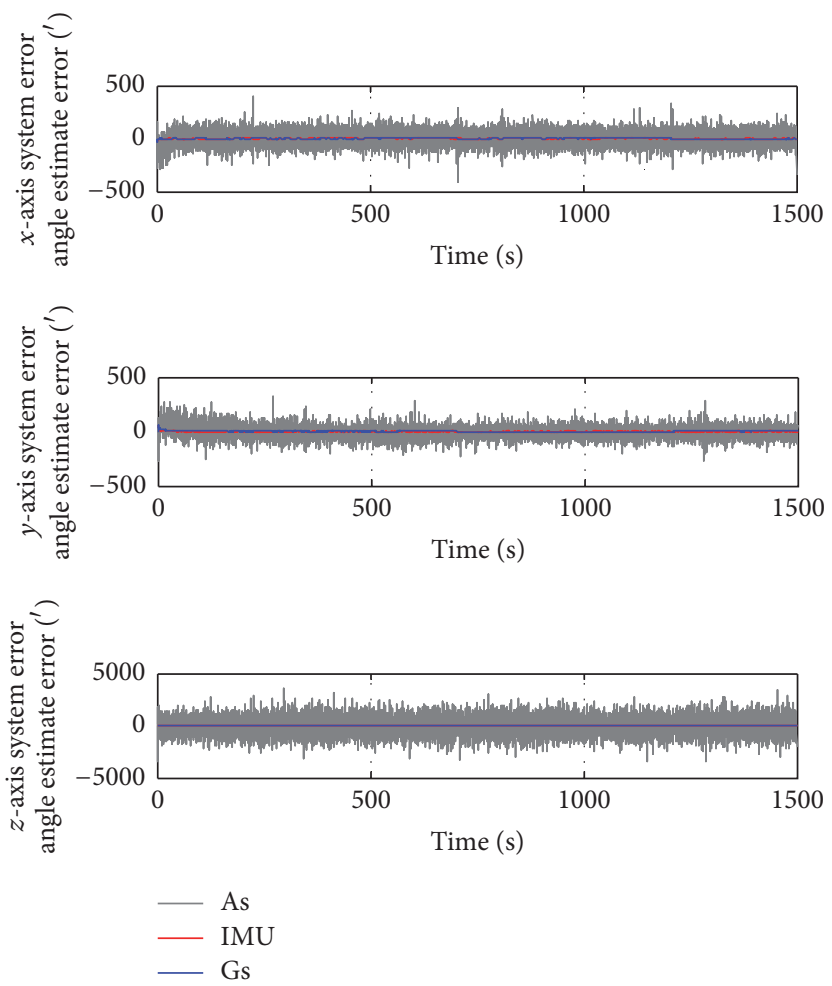

FIGURE 13: System error angle estimate error in semiphysical simulation. 
TABLE 4: Estimate errors of system error angle in experiment test $\left(^{\prime}\right)$.

\begin{tabular}{|c|c|c|c|c|c|c|}
\hline \multirow{2}{*}{ Errors } & \multicolumn{3}{|c|}{ L1 (300s-700s) } & \multicolumn{3}{|c|}{ L2 (800s-1200s) } \\
\hline & IMU & Gs & As & IMU & Gs & As \\
\hline \multicolumn{7}{|l|}{ STD } \\
\hline$x$-axis & 0.5469 & 0.8500 & 42.9655 & 0.5124 & 0.5549 & 42.7889 \\
\hline$y$-axis & 0.2256 & 0.4333 & 27.5946 & 0.2340 & 0.3020 & 27.4013 \\
\hline$z$-axis & 1.5704 & 0.5979 & 647.4333 & 0.5919 & 0.8776 & 642.9184 \\
\hline \multicolumn{7}{|l|}{ RMSE } \\
\hline$x$-axis & 0.6640 & 0.9841 & 43.1235 & 0.5625 & 1.0232 & 42.9072 \\
\hline$y$-axis & 0.2401 & 0.5429 & 29.7259 & 0.2556 & 0.9420 & 29.2839 \\
\hline$z$-axis & 5.7615 & 2.0966 & 648.8067 & 0.6068 & 1.3364 & 644.4869 \\
\hline
\end{tabular}

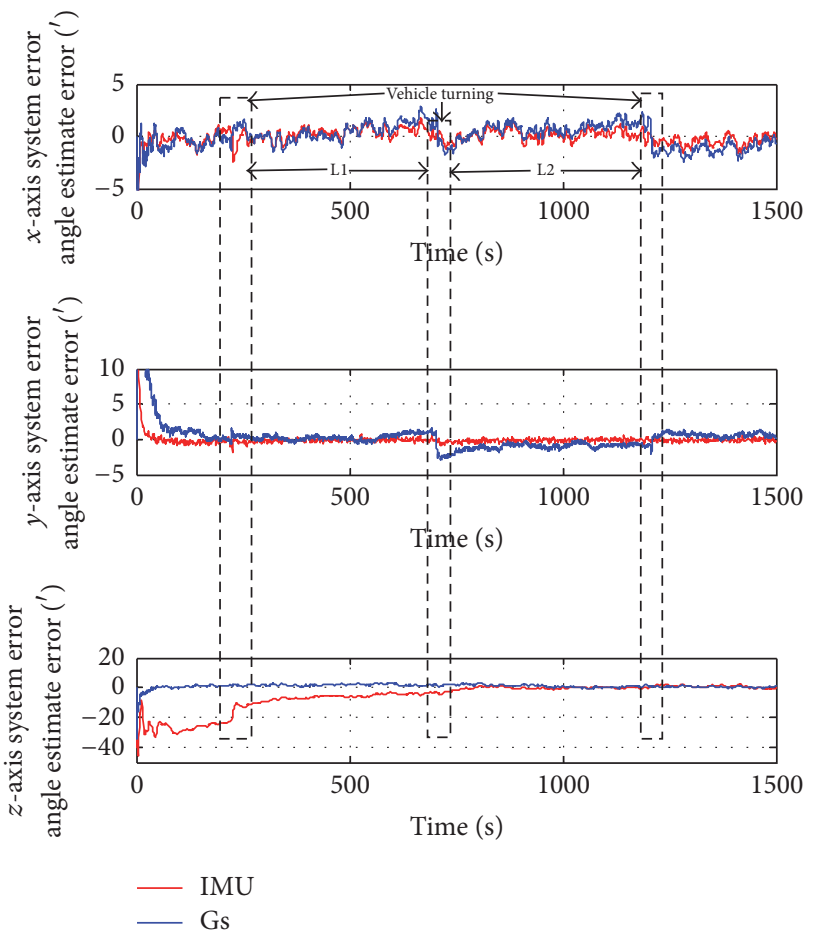

FIGURE 14: System error angle estimate error of methods based on IMU and Gs, respectively, in semiphysical simulation.

limitation on the weight, size, and cost of the measurement equipment. In view of the deformation measuring method based on Gs and As, the intrinsic relationship between the maneuver and the estimation accuracy needs further analysis and research in the future.

\section{Conflicts of Interest}

The authors declare that there are no conflicts of interest regarding the publication of this paper.

\section{Acknowledgments}

This work was supported in part by the National Natural Science Foundation of China (Grants nos. 61473020,
61421063, and 61233005), by the National High Technology Research and Development Program of China (863 Program) (Grants nos. 2015AA124001 and 2015AA124002), and by the International (Regional) Cooperation and Communication Project (Grant no. 61661136007).

\section{References}

[1] W. Xu, Y. Deng, and R. Wang, "Multichannel synthetic aperture radar systems with a planar antenna for future spaceborne microwave remote sensing," IEEE Aerospace and Electronic Systems Magazine, vol. 27, no. 12, pp. 26-30, 2012.

[2] P. Scherbaum, J. Brauchle, T. Kraft, and S. Pless, "MACS-Mar - A Real-Time Capable Multisensor Remote Sensing System for Maritime Applications: Current State of Development," in Proceedings of the IEEE International Conference on Aerospace Electronics and Remote Sensing Technology, ICARES 2015, Bali, Indonesia, 2015.

[3] Q. Zhang, X. J. Niu, Q. J. Chen, H. P. Zhang, and C. Shi, "Using Allan Variance to Evaluate the Relative Accuracy on Different Time Scales of GNSS/INS Systems," Measurement Science and Technology, vol. 24, no. 8, 2013.

[4] L.-Z. Chen and J. Fang, "A hybrid prediction method for bridging GPS outages in high-precision POS application," IEEE Transactions on Instrumentation and Measurement, vol. 63, no. 6, pp. 1656-1665, 2014.

[5] J. T. Case, M. T. Ghasr, and R. Zoughi, "Correcting mutual coupling and poor isolation for real-time 2-D microwave imaging systems," IEEE Transactions on Instrumentation and Measurement, vol. 63, no. 5, pp. 1310-1319, 2014.

[6] J. Wang, L. Y. Chen, X. D. Liang et al., "Multi-input Multi-output Frequency-modulated Continuous Wave Synthetic Aperture Radar System using Beat-frequency Division Waveforms," Measurement Science and Technology, vol. 24, no. 7, Article ID 074016, 2013.

[7] A. Liu and H. X. Ma, "Notice of Retraction Optimization Method for the Layout of IMUs in Deformation Detection System of Warship Based on Genetic Algorithm," in Proceedings of the 6th International Conference on Natural Computation (ICNC), vol. 8, 155, pp. 4014-4017, Shandong, China, 2010.

[8] R. C. Zheng, G. L. Yang, C. Y. Chen, and H. N. Weng, "Layout Optimization of Shipborne IMU Based on Genetic Algorithm," Journal of Chinese Inertial Technology, vol. 15, no. 2, pp. 177-280, 2007. 
[9] H. Dai, A. Liu, J. Lu, S. Dai, X. Wu, and Y. Sun, "Optimization about the layout of IMUs in large ship based on fruit fly optimization algorithm," International Journal for Light and Electron Optics, vol. 126, no. 4, pp. 490-493, 2015.

[10] T. H. Skopinski, W. S. Aiken, and W. B. Huston, "Calibration of Strain-gage Installations in Aircraft Structures for the Measurement of Flight Loads," NACA-Report-1178, Technical Report Archive and Image Library, 1954, pp. 505-536.

[11] J. M. Jenkins and V. M. DeAngelis, "A Aummary of Numerous strain-gage Load Calibrations on Aircraft Wings and Tails in A Technology Format," Nasa Technical Memorandum 4804, Dryden Flight Research Center, Edwards, Calif, UAS, 1997.

[12] A. Lizotte and W. Lokos, "Deflection-based aircraft structural loads estimation with comparison to flight," in Proceedings of the 46th AIAA/ASME/ASCE/AHS/ASC Structures, Structural Dynamics and Materials Conference, pp. 1-18, Austin, Tex, USA, 2005.

[13] V. De Angelis, "HiMAT flight deflection measurements," in Proceedings of the NASA HiMAT Symposium Paper, 1984.

[14] V. M. De Angelis, "In-flight deflection measurement of the HiMAT aeroelastically tailored wing," Journal of Aircraft, vol. 19, no. 12, pp. 1088-1094, 1982.

[15] H. Kannemans, "Flight testing of a wing deflection measurement method," in Proceedings of the 20th Atmospheric Flight Mechanics Conference, Baltimore, Md, USA, 1995.

[16] H. P. J. Veerman, H. Kannemans, and H. W. Jentink, "High accuracy in-flight wing deformation measurements based on optical correlation technique," in Proceedings of the 19th SFTE (EC) Symposium, Manching, Germany, 2008.

[17] J. T. Black, N. A. Pitcher, M. F. Reeder, and R. C. Maple, "Videogrammetry dynamics measurements of a lightweight flexible wing in a wind tunnel," Journal of Aircraft, vol. 47, no. 1, pp. 172180, 2010.

[18] S. A. Frost, C. H. G. Wright, R. W. Streeter, M. A. Khan, and S. F. Barrett, "Bio-mimetic optical sensor for structural deflection measurement," in Proceedings of the Bioinspiration, Biomimetics, and Bioreplication, San Diego, Calif, USA, March 2014, 9055:90550A.

[19] M. Scheerer, Z. Djinovic, and M. Schüller, "Fiber optic system for deflection and damage detection in morphing wing structures," in Proceedings of the 2013 SPIE Conference on Sensors and Smart Structures Technologies for Civil, Mechanical, and Aerospace Systems 2013, March 2013.

[20] W. Wu, S. Chen, and S. Qin, "Online estimation of ship dynamic flexure model parameters for transfer alignment," IEEE Transactions on Control Systems Technology, vol. 21, no. 5, pp. 1666-1678, 2013.

[21] J. H. Cheng, T. D. Wang, D. X. Guan, and M. L. Li, "Polar transfer alignment of shipborne SINS with a large misalignment angle," Measurement Science and Technology, vol. 27, no. 3, Article ID 035101, 2016.

[22] R. E. Kalman, "A new approach to linear filtering and prediction problem," Journal of Basic Engineering, vol. 82, no. D, pp. 35-45, 1960.

[23] S. Eichstädt, N. Makarava, and C. Elster, "On the evaluation of uncertainties for state estimation with the kalman filter," Measurement Science and Technology, vol. 27, no. 12, 1968.

[24] X. Gong, R. Zhang, and J. Fang, "Application of unscented RT-S smoothing on INS/GPS integration system post processing for airborne earth observation," Measurement: Journal of the International Measurement Confederation, vol. 46, no. 3, pp. 1074-1083, 2013.
[25] M. S. Grewal, L. R. Weill, and A. P. Andrews, Global Positioning Systems, Inertial Navigation, and Integration, John Wiley \& Sons, Inc., Hoboken, NJ, USA, 2007.

[26] S. Nassar, Improving the Inertial Navigation System (INS) Error Model for INS and INS/DGPS Applications [Ph.D. thesis], Department of Geomatics Engineering, University of Calgary, Canada, 2003.

[27] M. Y. Zhong, Q. Cao, J. Guo, and D. H. Zhou, "Simultaneous lever-arm compensation and disturbance attenuation of POS for a UAV surveying system," IEEE Transations on Instrumentation Measurement, vol. 65, no. 12, pp. 2828-2839, 2016.

[28] X. M. Wang and W. B. Ni, "An Improved Particle Filter and Its Application to an INS/GPS Integrated Navigation System in a Serious Noisy Scenario," Measurement Science and Technology, vol. 27, no. 9, p. 2016, 2016.

[29] M. Y. Zhong, J. Guo, D. F. Guo, and Z. H. Yang, "An Extended $H_{i} / H_{\infty}$ Optimization Approach to Fault Detection of INS/GPSintegrated System," IEEE Transations on Instrumentation Measurement, vol. 65, no. 11, pp. 2495-2504, 2016.

[30] Y. Lu and X. Cheng, "Random misalignment and lever arm vector online estimation in shipborne aircraft transfer alignment," Measurement: Journal of the International Measurement Confederation, vol. 47, no. 1, pp. 756-764, 2014.

[31] D. Li, M. Zhong, and D. Guo, "Error Detection and Compensation in Transfer Alignment for the Distributed POS," in Proceedings of the 25th Chinese Control and Decision Conference, pp. 25-27, Guiyang, China, 2013.

[32] Z. Feng, W. Jiang, and S. Gao, "Research of rapid transfer alignment and observability analysis," in Proceedings of the 2nd International Conference on Intelligent Control and Information Processing, ICICIP 2011, pp. 204-207, Harbin, China, 2011.

[33] "Novatel OEMV-3 receiver board," http://www.novatel.com.cn/ products/gnss-receivers/oem-receiver-boards/oemv-receivers/. 


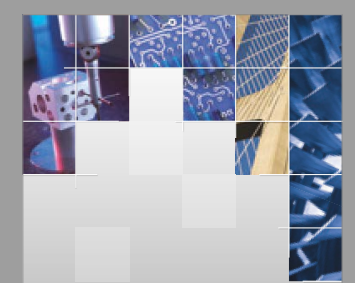

\section{Enfincering}
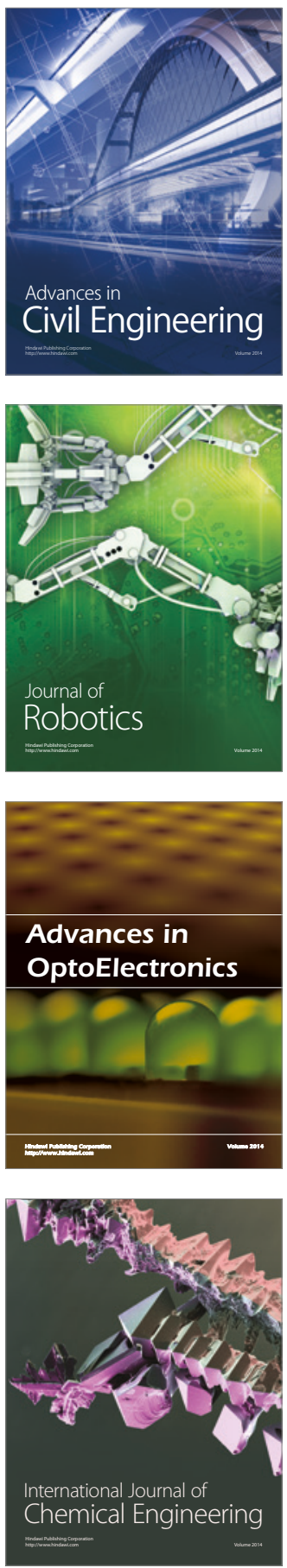

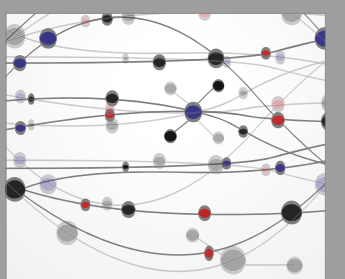

The Scientific World Journal

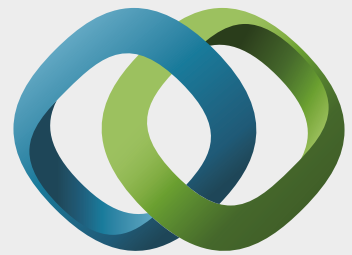

\section{Hindawi}

Submit your manuscripts at

https://www.hindawi.com
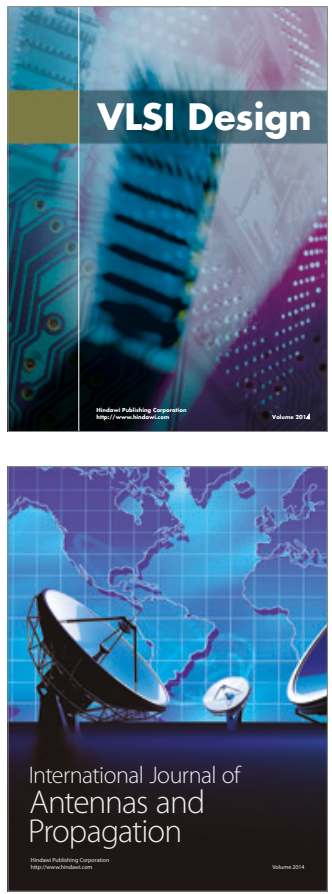

\section{Rotating}

Machinery
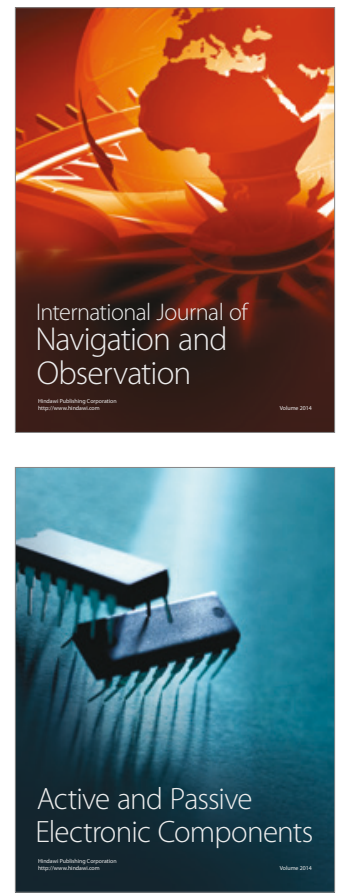
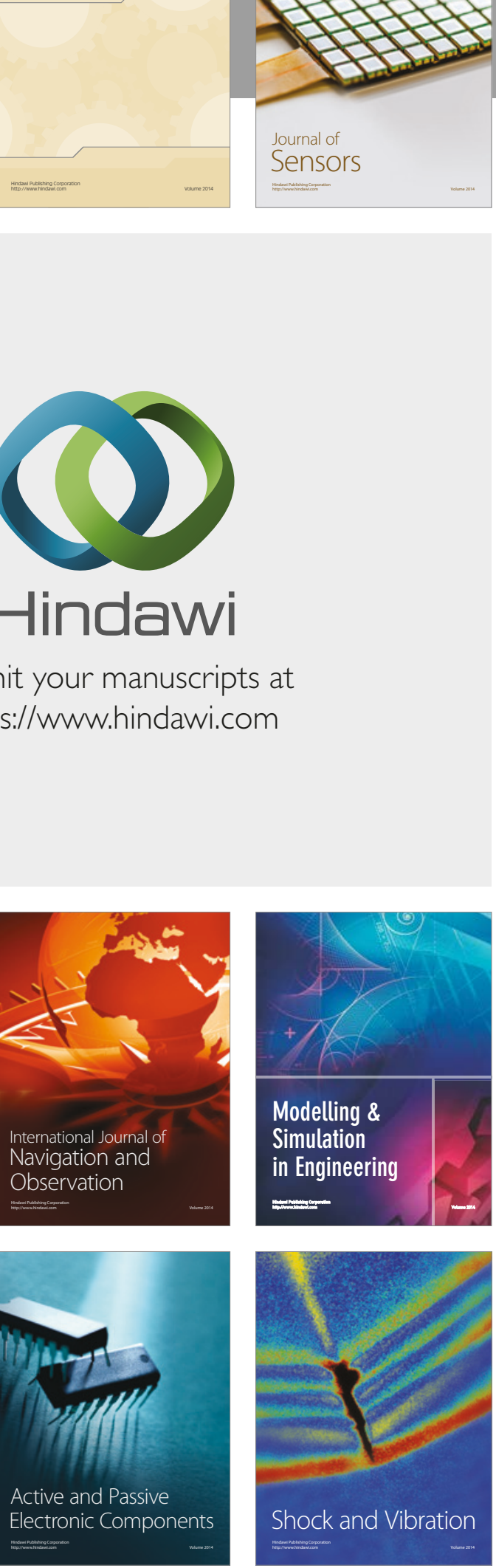
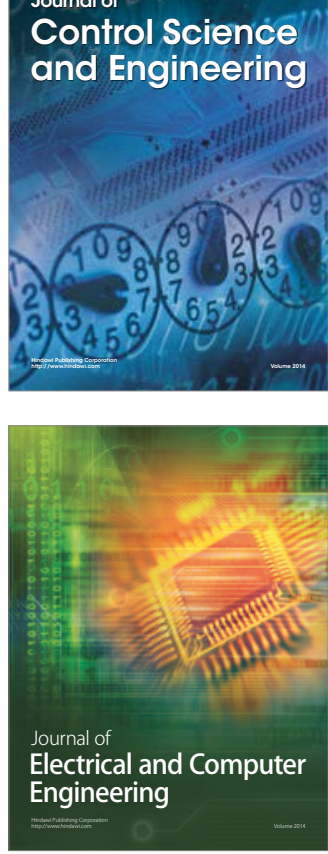

Distributed

Journal of

Control Science

and Engineering
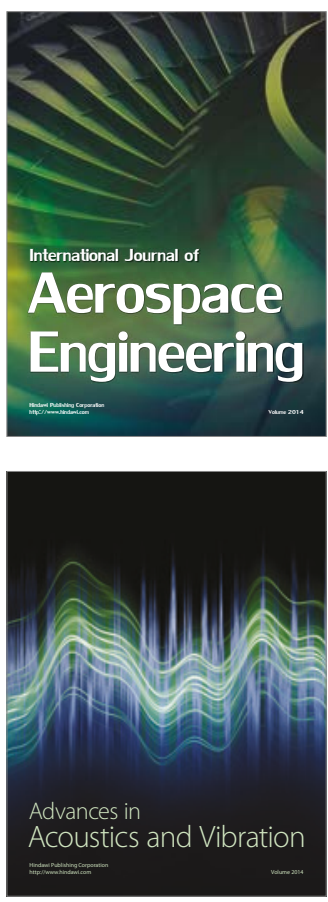

Sensor Networks 